\title{
Extracción y clasificación de características de imágenes de incendios forestales
}

\author{
Belém Priego Sánchez ${ }^{1}$, Esteban Castillo ${ }^{2}$, Arturo Bustamante ${ }^{3}$, Iván Olmos ${ }^{3}$ \\ ${ }^{1}$ Laboratoire LDI (Lexique, Dictionnaires, Informatique) \\ Université Paris 13, Sorbonne Paris Cité, Villetaneuse, France \\ belemps@gmail.com \\ ${ }^{2}$ Universidad de las Américas Puebla \\ Departamento de Computación, Electrónica y Mecatrónica, México \\ esteban.castillojz@udlap.mx \\ ${ }^{3}$ FCC, Benemérita Universidad Autónoma de Puebla, México \\ $\{$ dvartus, ivanoprkl\}@gmail.com
}

\begin{abstract}
Resumen Este artículo se enfoca en el análisis y extracción de características de imágenes de incendios forestales (usando procesamiento digital de imágenes), las cuales son utilizadas como entrada en un proceso de clasificación para la construcción de modelos que permitan a futuro identificar si una imagen tiene o no un incendio forestal. Las imágenes se procesaron a través de un proceso de particionamiento, donde de cada fragmento se obtuvieron un conjunto de características basadas en la estadística. Para el proceso de clasificación, se utilizaron dos técnicas de aprendizaje automático: árboles de decisión y redes neuronales. A partir de los resultados obtenidos, se logró obtener una clasificación global del $84 \%$, mostrando que el proceso de particionamiento de la imagen, junto con los clasificadores seleccionados, son un medio viable para identificar incendios forestales a partir de fotografías digitales.
\end{abstract}

Palabras clave: Incendios forestales, minería de datos, árboles de decisión, red neuronal.

\section{Introducción}

Los incendios forestales son uno de los fenómenos que afectan negativamente tanto a la naturaleza como a la calidad de vida de los humanos. Desde el punto de vista ecológico, un incendio forestal altera un ecosistema, desde el tipo de vegetación presente en la zona siniestrada, hasta la fauna que habita en ella.

En México, existen esfuerzos tanto a nivel federal como estatal para detectar de forma temprana un incendio forestal. Por ejemplo, en el estado de Puebla, desde el año 2009 se instaló una red de videocámaras que permiten monitorear regiones del estado de Puebla propensas a estos fenómenos. El sistema es operado de forma remota por personal de la Secretaría de Medio Ambiente y Recursos Naturales (SMNR) a través de un centro de control ubicado en la reserva 
ecológica Flor del Bosque. A pesar de ser un importante avance en el combate de los incendios forestales, este sistema tiene algunos puntos de oportunidad que pueden ser mejorados. Uno de ellos es la manipulación manual para la detección de los incendios forestales, ya que requiere que el personal manipule manualmente cada videocámara en busca de un posible incendio forestal, lo cual puede llegar a ser cansado y tedioso.

Una estrategia que puede resolver este problema planteado es el desarrollo de un sistema que permita procesar imágenes de incendios forestales y que determine de forma automática la presencia de estos eventos en una región. En este sentido, en el presente trabajo se propone un método para el procesamiento de las imágenes, que permita clasificar a nuevas imágenes como dos clases: presencia de incendio forestal o ausencia del mismo.

La propuesta se basa en el proceso de Descubrimiento de Conocimiento en Bases de Datos (Proceso KDD), que contempla desde la construcción de una base de datos (en este caso, imágenes con incendios forestales), selección de las muestras más representativas, proceso de transformación, técnicas de aprendizaje automático para aprender las características de los patrones (los incendios forestales), hasta la interpretación de los resultados. En este trabajo, la parte de aprendizaje automático se centró en árboles de clasificación (decisión) y redes neuronales, que son técnicas que permiten construir modelos descriptivos para la clasificación de instancias, en donde es posible analizar y construir reglas para entender que características influyen en el proceso de clasificación [1]. Para esto se propone utilizar un proceso de análisis de las imágenes basado en particiones, con el fin de medir con mayor precisión las caracterticas propias de un incendio forestal.

Un incendio forestal (IF) es la propagación libre y no programada del fuego sobre la vegetación, causado en forma natural, accidental o intencional debido a lo cual se afectan combustibles naturales situados en áreas boscosas [2]. Existen varios tipos de incendios, los más comunes en México son los superficiales en un $90 \%$ [3]. Esta clase de desastre se caracteriza porque el fuego consume únicamente lo que los especialistas llaman combustibles forestales, es decir: hierbas, sácate, hojarasca y leña suelta; sin dañar los árboles en su totalidad en algunos casos.

En investigaciones sobre incendios forestales, la propagación del fuego depende de la húmedad, de la acumulación y combinación de los atributos de los combustibles. En las hierbas, arbustos, árboles vivos la propagación depende de su humedad. Por otro lado, se encuentran las ramas, hojas y arbustos secos que tienen alta inflamabilidad dependiendo de su abundancia y posición (generalmente en el suelo) por lo que también se les llama combustibles rápidos.

A partir de 1998, en México el Servicio Meteorológico Nacional empezó a intentar reducir los problemas causados por los IF [4], debido a que el número de incendios se incremento de manera alarmante, la medida que se tomó es la creación del Programa para Detección de Puntos de Calor Mediante Técnicas de Percepción Remota [5], cuyo propósito principal es ubicar aquellos sitios donde se registran temperaturas mucho más altas de lo normal, a fin de alertar 
sobre posibles incendios [5]. En este programa se uso imágenes de satélite y en particular, las generadas por el sensor AVHRR (Advanced Very High Resolution Radiometer) de la Nacional Oceanic and Atmospheric Administration (NOAA).

El Servicio Meteorológico Nacional cuenta con un centro de monitoreo, en el que reciben imágenes de todo el territorio mexicano, generadas pos dos satélites estadounidenses: Terra y Aqua, mismos que proporcionan diariamente 8 reportes, 4 en el día y 4 en la noche [6], la detección se realiza principalmente a través de imágenes obtenidas del sensor NOAA/16-AVHRR que proporciona 5 bandas para cada pixel, los algoritmos para el procesamiento de imágenes fueron desarrollados por la NASA y la universidad de Winsconsin-CIMSS.

Cabe mencionar que los satélites de órbita polar como el NOAA tienen una amplia resolución espacial [4], pero tienen el inconveniente de proporcionar ciclos limitados con sólo 1 o 2 pasadas por sobre el mismo lugar [4], la alternativa a este tipo de satélites es la utilización de estos como el Geostationary Operational Environmental Satellite (GOES), que proporciona un ciclo con mayores recorridos sobre el mismo lugar con el inconveniente de que la resolución espacial es de mucho menor tamaño (apenas $4 \mathrm{~km}$.) [4].

Si bien los modelos mencionados anteriormente permiten en gran medida la detección de IF, aún presentan grandes desventajas como son: la dependencia de satélites estadounidenses, esto representa la desventaja de que solamente 8 imágenes son proporcionadas cada 24 horas, aunque el trabajo con satélites GOES [4] permite reducir los tiempos de espera entre cada muestra sobre un mismo punto, aun se presenta la desventaja de que los resultados son confiables solo si se cuenta con la ausencia de nubes además para imágenes nocturnas se debe de tener en cuenta el no confundir la iluminación de las ciudades con un incendio forestal.

Las desventajas anteriormente mencionadas han motivado a la utilización de otros tipos de sistemas que no utilicen imágenes provenientes de sensores de satélites, como es el caso del Sistema de Información para el Monitoreo de Incendios Forestales (SIMIF) en el estado de Puebla. Éste sistema se basa en el uso de cámaras para el monitoreo de bosques, permitiendo que estas zonas estén vigiladas las 24 horas del día. Con este tipo de sistemas se pueden obtener imágenes a intervalos de tiempo que no dependan del clima.

Como parte de la búsqueda de conocimiento dentro del área de minería de datos así como la teoría relacionada a la prevención de incendios, podemos encontrar múltiples referencias a trabajos relacionados al planteado en este artículo, dichos trabajos muestran una fuerte relación a los objetivos buscados, los cuales son encontrar características que nos permitan reducir o eliminar la posibilidad de incendios forestales, por lo que a continuación se presentan algunos trabajos relevantes. OIlero [7] presenta un sistema multi-sensorial para la detección temprana de incendios forestales, se emplean principalmente imágenes infrarrojas. Yasar [8] utiliza animales como sensores biológicos móviles para hacer la detección temprana de incendios forestales. Florent [9] propone un método totalmente automático de detección de incendios forestales a partir de imágenes satélitales sobre la base de la TIR (Thermical InfraRed). González, Hernández 
y Jiménez [10], realizan un sistemas que incorpora autómatas celulares para la detección del comportamiento de los incendios forestales en un área determinada. Seng, Wynne y Kim [11], presentan un enfoque para reducir la falsas alarmas en los puntos calientes de las regiones de los incendios forestales, emplea las coordenadas geográficas de calor asi como manchas en las regiones de los incendios forestales en la detección probable de los puntos de incendio. Con el fin de determinar los patrones regulares en los derivados puntos de acceso y los clasifique como las falsas alarmas en el supuesto de que los incendios por lo general no se propagan en patrones regulares, como una línea recta.

El resto de este trabajo está estructurado de la siguiente manera: En la Sección 2 se explica con detalle la propuesta que se llevó a cabo para la realización de este trabajo, obteniendo resultados que se muestran en la Sección 3 y finalmente reportando conclusiones en la Sección 4 y con base en estas conclusiones se introduce el posible trabajo a futuro en esta misma sección.

\section{Metodología propuesta}

El objetivo de este trabajo es el de identificar y clasificar imágenes de incendios forestales, los pasos para la construcción de un modelo de clasificación se muestran en la figura 1. Básicamente consiste en construir una base de datos, ésta contiene la mayor cantidad de datos posibles, una vez teniendo los datos es necesario seleccionar los que realmente servirán para el objetivo planteado, transformándolos como entrada al modelo de clasificación, en este caso: árboles de clasificación y redes neuronales, para posteriormente evaluar los resultados y descubrir conocimiento.

En las siguientes secciones se describe a detalle cada una de las etapas del proceso aplicado.

\subsection{Selección de los datos}

Las imágenes utilizadas tienen la finalidad de proporcionar una similitud con las imágenes provenientes de las cámaras del SIMIF, esto añade como ventaja poder aplicar esta metodología no únicamente con cámaras del SIMIF si no que podrá ser utilizada por otros sistemas que trabajen con imágenes de características similares.

Para la construcción de la base de datos, se hizo necesaria la ayuda del gran repositorio que es la Web. Es decir, se obtuvieron la mayor cantidad de imágenes de incendios forestales que estuvieran disponibles en la Web mediante una consulta a un buscador. Con la finalidad de obtener una amplia colección de distintos tipos de incendios y diferentes perspectivas, con el propósito de mejorar la detección de incendios.

Por el momento, no importaba si la imagen tenía un retoque, si tenía o no un incendio forestal o si simplemente el buscador nos la proporcionaba como resultado. En la tabla 1 se describen las características de las imágenes de incendios forestales recolectadas de la Web. El objetivo de la recolección era 


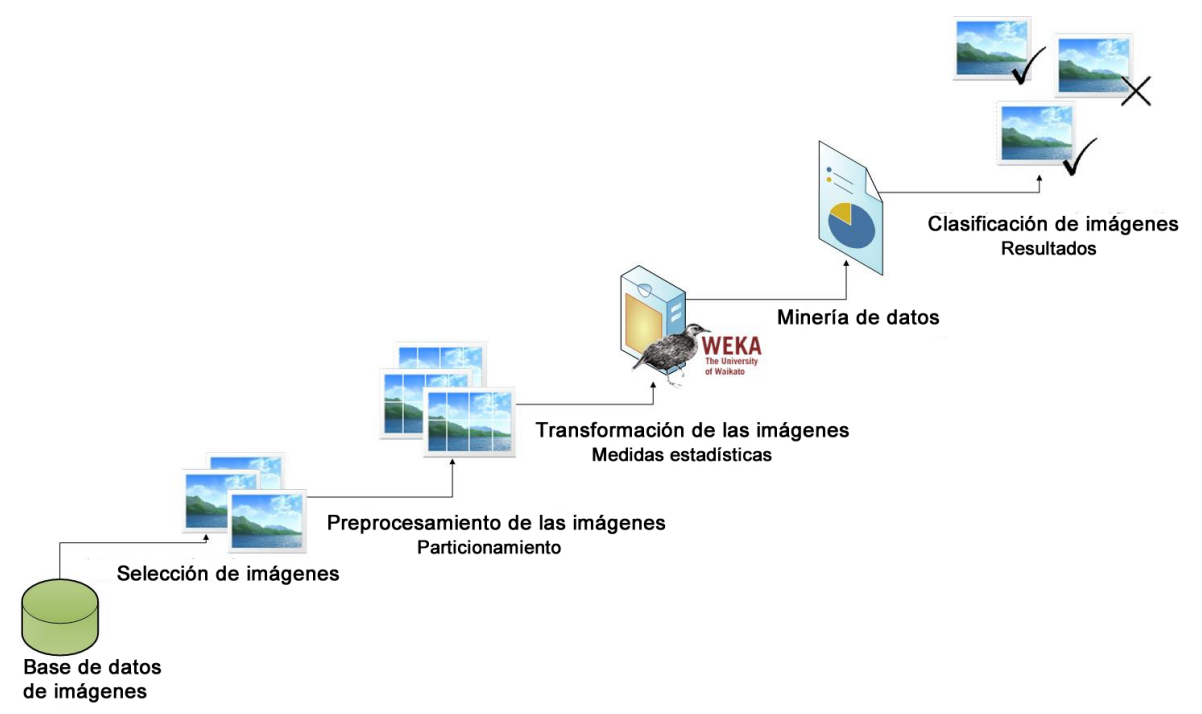

Fig. 1. Metodología para la construcción de un modelo de clasificación

tener una gran base de datos de imágenes donde estuviera inmerso un incendio forestal, que en una etapa posterior se seleccionaran las imágenes que realmente se ocuparán.

Tabla 1. Características de las imágenes de incendios forestales recolectadas de la Web

\begin{tabular}{|c|c|}
\hline Característica & Descripción \\
\hline Número de imágenes recolectadas & 535 \\
\hline Imágenes con incendios de día & 198 \\
\hline Imágenes con incendios de noche & 120 \\
\hline Imágenes solamente con humo & 100 \\
\hline Imágenes sin visualización de fuego o humo & 117 \\
\hline Formato de la imagen & JEPG \\
\hline Resolución promedio & $400 \times 400$ \\
\hline
\end{tabular}

\subsection{Preprocesamiento de los datos}

De las imágenes descargadas de la Web, se prosiguió a identificar manualmente las que realmente servirían para la tarea de identificación de incendios forestales ocupando para el propósito planteado una muestra de 98 imágenes que proporcionan información para la identificación de un incendio forestal y que constan de las características descritas en la tabla 2. 
Una vez obtenida la muestra, se continuó con el particionamiento de las imágenes y este se realizó utilizando la propiedad Quadtree mediante un software extractor de características que fué implementado. El quadtree permite hacer operaciones sobre las imágenes, así como unirlas, modificar ciertos cuadrantes de la imagen, agregar/modificar colores, entre otras. Dentro de los tipos de quadtree podemos encontrar los siguientes: quadtree de puntos, quadtree de región y quadtree de cubo.

Tabla 2. Características de las imágenes de incendios forestales seleccionadas

\begin{tabular}{|c|c|}
\hline Característica & Descripción \\
\hline Número de imágenes seleccionadas & 98 \\
\hline Imágenes con incendios de día & 49 \\
\hline Imágenes con incendios de noche & 49 \\
\hline Formato de la imagen & JEPG \\
\hline Resolución promedio & $400 \times 400$ \\
\hline Número de particiones de la imagen & 8 \\
\hline Distribución de las particiones & $100 \times 200$ \\
\hline
\end{tabular}

De los cuales el quadtree de región es el más importante en el procesamiento digital de imágenes y es el tipo de quadtree que se utilizó para particionar la imagen. El quadtree de región representa una partición del espacio en dos dimensiones descomponiendo la región en cuatro cuadrantes iguales, subcuadrantes, y así sucesivamente con cada nodo de la hoja que contiene los datos que corresponden a una subregión específica.

Es importante mencionar que para cada partición se seleccionó de manera no automática, si dicha partición tiene fuego o no, esta característica es almacenada en la base de información como el atributo clasificador $(C)$. Para obtener el atributo clasificador de la imagen que fue dividida en 8 particiones, se aplican las siguientes reglas:

1. Si cualquiera de las particiones obtiene un resultado positivo(por medio del software generado), entonces la imagen(las 8 particiones) se considerara como positiva

2. Si ninguna de las 8 particiones obtiene un resultado positivo, entonces la imagen real se considerara como negativa

En la figura 2 se muestra un ejemplo del proceso anteriormente descrito para la obtención de una muestra positiva y una muestra negativa.

\subsection{Transformación de los datos}

La obtención de resultados implica la creación de nuevo conocimiento por medio de información previamente adquirida en este caso, propiedades de imágenes 

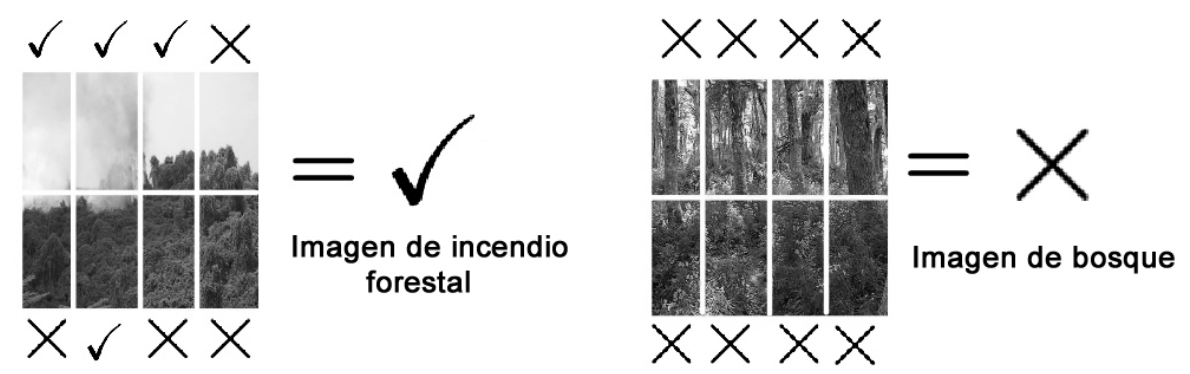

Fig. 2. Ejemplo de clasificación positiva y negativa.

de incendios forestales, para obtener dichas propiedades de manera automática, se creó un sistema extractor de características el cual permite abstraer de cada imagen, características de tipo estadísticas y de textura las cuales son usadas después por algoritmos de minería de datos, donde dicho software genera por cada imagen un quadtree de 8 elementos, de cada elemento de este quadtree se obtiene una tupla de información, abstrayendo la información de ésta y almacenándola en una base de información.

Las técnicas para la extracción de características de las imágenes, son de tipo estadístico, están basadas en el uso de histogramas $p_{f}(f)$ donde dejemos que $f_{k}$, $k=1, \ldots, N$ sea los niveles de intensidad de la imagen. Las medidas de textura basadas en el histograma son las siguientes:

- Varianza de textura $(\sigma)$ : Medida de la dispersión de las intensidades de las regiones.

$$
\sigma^{2}=\sum_{k=1}^{N}\left(f_{k}-\mu\right)^{2} p_{f}\left(f_{k}\right)
$$

- Kurtosis $\left(\mu_{4}\right)$ : Medidad de la cola del histograma, donde histogramas con una cola considerablemente larga corresponden a regiones con altos picos de concentración de información.

$$
\mu_{4}=\frac{1}{\sigma^{4}} \sum_{k=1}^{N}\left(f_{k}-\mu\right)^{4} p_{f}\left(f_{k}\right)-3
$$

- Media $(\mu)$ : Es una estimación del nivel de intensidad en la región.

$$
\mu=\sum_{k=1}^{N} f_{k} p_{f}\left(f_{k}\right)
$$

- Oblicuidad(skewness) $\left(\mu_{3}\right)$ : Medida de la simetría del histograma, la cual muestra el porcentaje de las regiones de pixeles que favorecen las intensidades.

$$
\mu_{3}=\frac{1}{\sigma^{3}} \sum_{k=1}^{N}\left(f_{k}-\mu\right)^{3} p_{f}\left(f_{k}\right)
$$


- Entropía $(H)$ : Medida que expresa el grado de incertidumbre que existe sobre un conjunto de datos de los cuales sólo interesa alguno en particular.

$$
H=-\sum_{k=1}^{N} p_{f}\left(f_{k}\right) \ln p_{f}\left(f_{k}\right)
$$

\subsection{Minería de datos}

Tomando la herramienta $\mathrm{WEKA}^{1}$ como el software extractor de conocimientos, se definió como serán las tuplas (en la base de datos), usando como atributos a las funciones que se definieron en la sección 2.3 como se muestra en la figura 3 .

$$
\underbrace{(M 1, M 2, M 3, I}_{\text {Media }} \underbrace{D 1, D 2, D 3, I}_{\text {Desviacion }} \underbrace{E 1, E 2, E 3, I}_{\text {Entropia }} \underbrace{V 1, V 2, V 3, I}_{\text {Varianza }} \underbrace{S 1, S 2, S 3, I}_{\text {Skewness }} \underbrace{K 1, K 2, K 3, I}_{\text {Kurtosis }}, C)
$$

Fig. 3. Representación vectorial utilizada.

De dicha formula se puede notar que cada medida se usa para cada canal del RGB, donde los valores van de 0 a 255 , así como se puede denotar el valor $I$ el cual se define como la intensidad o en otras palabras como la suma de los tres canales, éste nos muestra las características globales de la imagen. Finalmente se muestra el atributo $C$ el cual es el atributo clasificador y puede tener los valores de positivo o negativo.

De todos los métodos de aprendizaje (o de obtención del conocimiento) los sistemas de aprendizaje basados en árboles de clasificasión son quizás el método más fácil de utilizar y de entender. Un árbol de clasificasión es un conjunto de condiciones organizadas en una estructura jerárquica, de tal manera que la decisión final se puede determinar siguiendo las condiciones que se cumplen desde la raíz hasta alguna de sus hojas. Una de las grandes ventajas de los árboles de clasificasión es que, en su forma más general, las opciones posibles a partir de una determinada condición son excluyentes. Ésto permite analizar una situación y, siguiendo el árbol de clasificasión apropiadamente, llegar a una sola acción o decisión a tomar [12].

En el caso del árbol de clasificasión se utilizó el algoritmo C4.5 genera un árbol de clasificación a partir de los datos mediante particiones realizadas recursivamente. El árbol se construye mediante la estrategia de profundidad-primero (depth-first) [12].

Las redes neuronales son modelos que intentan reproducir el comportamiento del cerebro. Como tal modelo, realiza una simplificación, averiguando cuáles son los elementos relevantes del sistema, bien porque la cantidad de información de

\footnotetext{
${ }^{1}$ Consultar: http://www.cs.waikato.ac.nz/ml/weka/
} 
que se dispone es excesiva o bien porque es redundante. Una elección adecuada de sus características, más una estructura conveniente, es el procedimiento convencional utilizado para construir redes capaces de realizar una determinada tarea.

De manera concreta un perceptrón consiste en dos tipos de nodos: nodos de entrada, los cuales son usados para representar las entradas de los atributos, y los nodos de salida, los cuales son usados para representar el modelo de salida. Un perceptrón es conectado vía un enlace con peso a los nodos de salida. El enlace con peso es usado para emular la fuerza de una conexión sináptica entre neuronas. Así como en los sitemas biológicos de neuronas, el entrenamiento de un perceptrón le permite a éste adaptarse hasta que las salidas son consistentes con la verdadera salida de los ejemplos del set de entrenamiento [13].

\section{Resultados}

El proceso usado para obtener conocimiento de un conjunto de imágenes de incendio forestal, fué en principio particionar cada imagen en 8 Quadtree, donde de estas particiones se obtuvo un conjunto de tuplas(una por cada partición de imagen) por medio de un sistema extractor de características, teniendo dichas tuplas se experimentó primero con un árbol de decisión usando el algoritmo $\mathrm{C}$ 4.5, donde principalmente se usó una validación cruzada de 10 elementos y se cambiaron las propiedades de ejecución del algoritmo, cambiando las propiedades más importantes. En el caso de una red neuronal, se usó un perceptrón multicapa, ésto es, múltiples secciones o capas cada una con un conjunto de neuronas las cuales se conectan unas con otras para obtener un mejor aprendizaje, así como se eligió una función de transferencia sigmoidal, y al igual que con un árbol de decisión se hicieron pruebas cambiando el número de neuronas y de capas así como las opciones principales sobre el algoritmo.

En la tabla 3 se muestran los porcentajes de clasificación de instancias correctamente clasificadas (Instancias C.C) que se obtuvieron al aplicar árboles de decisión usando el algoritmo C4.5.

Tabla 3. Resultados al aplicar Árboles de Decisión

\begin{tabular}{|c|c|c|c|c|c|}
\hline Instancias C.C & BinarySplits & MinNumObj & numFolds & ConfFactor & RedErrorPruning \\
\hline 78.699 & true & 2 & 3 & 0.25 & false \\
\hline 77.2959 & false & 6 & 3 & 0.25 & false \\
\hline $\mathbf{7 8 . 8 2 6 5}$ & true & $\mathbf{3}$ & $\mathbf{3}$ & $\mathbf{0 . 2 5}$ & false \\
\hline 77.9337 & true & 5 & 3 & 0.25 & false \\
\hline 78.3163 & true & 4 & 3 & 0.25 & true \\
\hline 75.6378 & true & 3 & 4 & 0.15 & true \\
\hline 75.8929 & false & 2 & 3 & 0.25 & false \\
\hline 78.8260 & true & 3 & 3 & 0.35 & false \\
\hline 78.2092 & true & 2 & 3 & 0.40 & false \\
\hline
\end{tabular}


En la tabla 4 se muestran los porcentajes de clasificación de Instancias C.C al usar una red neuronal tipo perceptrón.

Tabla 4. Resultados al aplicar Redes Neuronales

\begin{tabular}{|c|c|c|c|c|c|}
\hline Instancias C.C & HiddenLayer & TrainingTime & LearningRate & Momentum & ValThreshold \\
\hline 84.1837 & 2,2 & 700 & 0.3 & 0.2 & 20 \\
\hline 81.7602 & 1,1 & 500 & 0.3 & 0.2 & 20 \\
\hline 82.9082 & 4,4 & 500 & 0.3 & 0.2 & 20 \\
\hline 83.1633 & 4,4 & 1000 & 0.5 & 0.2 & 20 \\
\hline 82.1429 & 4,4 & 1500 & 0.3 & 0.2 & 20 \\
\hline 83.0357 & 15 & 500 & 0.3 & 0.5 & 20 \\
\hline 83.4184 & 15,15 & 500 & 0.3 & 0.2 & 25 \\
\hline $\mathbf{8 4 . 4 3 8 8}$ & $\mathbf{2 0}$ & $\mathbf{1 0 0 0}$ & $\mathbf{0 . 3}$ & $\mathbf{0 . 2}$ & $\mathbf{2 0}$ \\
\hline 81.8878 & 30 & 500 & 0.3 & 0.2 & 25 \\
\hline 83.2908 & 30,30 & 1000 & 0.4 & 0.2 & 20 \\
\hline 60.7143 & $7,7,7,7$ & 1000 & 0.1 & 0.2 & 20 \\
\hline
\end{tabular}

Teniendo los resultados de ambas series de experimentos (red neuronal y árbol de clasificación) se obtuvieron las clasificaciones parciales, las cuales ayudaran a obtener las clasificaciones totales (la clasificación de la imagen verdadera) por medio de los pesos ajustados en el caso de la red neuronal y el árbol de clasificación obtenido en el caso del algoritmo C 4.5, en la tabla 5 se muestra el procentaje de instancias correctamente clasificadas al realizar la clasificación total.

Tabla 5. Comparativa de Clasificación verdadera

\begin{tabular}{|c|c|}
\hline Tipo & Instancias C.C \\
\hline Red Neuronal & 89.795 \\
\hline Árbol Clasificación & 87.755 \\
\hline
\end{tabular}

Teniendo la clasificación real se hizo una evaluación de que método de aprendizaje tiene un mejor desempeño teniendo una evidencia dada. Al aplicar las técnicas de evaluación con un intervalo de confianza del $90 \%$, se obtiene como resultado que la red neuronal presenta una mejor clasificación con respecto al árbol de clasificaión y es estadísticamente nos acerca de manera más favorable al error verdadero. 


\section{Conclusiones y Trabajo a Futuro}

Los incendios forestales desempeñan un papel fundamental en la transformación de los paisajes, la sucesión de la vegetación, la degradación del suelo y calidad de aire; por tal motivo este trabajo se centró principalmente en la identificación de este fenómeno. Actualmente tratar de detectar incendios forestales se realiza de diferentes maneras y se ha convertido en una tarea primordial para el cuidado y protección del medio ambiente; la técnica que se presenta en este trabajo ayudará a reconocer de una forma automática esta catastrofe que ocurre en el medio ambiente y que muchas de las veces se podria evitar si se tomaran las medidas necesarias para prevenirlos.

Por otro lado, se creó un software capaz de obtener información estadística y de textura de un set de imágenes de incendios forestales, observando que usar un particionamiento (Quadtree) ayuda a mejorar la precisión de los clasificadores que se utilizaron al realizar un modelo de clasificaión obteniendo un $89.795 \%$ de instancias clasificadas correctamente para el caso de una red neuronal frente a un $87.755 \%$ para el árbol de clasificación. Concluyendo que la red neuronal aportó mayor información y mayor conocimiento. De acuerdo a estos resultados se tiene que de acuerdo a los falsos positivos se consideró que es mejor que el clasificador diga que hubo un incendio forestal(positivo) cuando en realidad no lo hubo(negativo) porque de esta manera no se tiene un porcentaje de clasificación incorrecto, de acuerdo a los datos procesados.

El trabajo se centró en la detección de incendios forestales basandose en imágenes descargadas de la Web que no pertenecen a ningún sistema de monitoreo, sin embargo, en un futuro se pueden agregar imágenes a la base de datos provenientes del sistema que se vaya a utilizar para mejorar la detección de IF. Además de que se pueden utilizar otros clasificadores para ver su comportamiento en esta tarea.

Agradecimientos. Este trabajo ha sido parcialmente apoyado por el Consejo Nacional de Ciencia y Tecnología - CONACYT referencia 218862/314461.

\section{Referencias}

1. José Hernández Oralio, María José Ramírez Quintana, C.F.R.: Introducción a la Minería de Datos. Prentice Hall, Madrid (2004)

2. SEMARNAT: Secretaría de medio ambiente y recursos naturales (2010) Dependencia de Gobierno Federal.

3. INIFAP: Instituto nacional de investigaciones forestales, agrícolas y pecuarias (2010) Institución de excelencia científica y tecnologíca.

4. Viller Ruiz Lourdes, L.B.J.: Incendios forestales en México. Métodos de evaluación. Universidad Nacional Autónoma de México, México (2004)

5. SEMARNAT: Cuenta méxico con un programa de detección que permite eficientar el combate a incendios forestales (2007) Secretaría de Medio Ambiente y Recursos Naturales. 
6. SMN: Detección de incendios forestales en méxico (2007) Servicio Meteorológico Nacional.

7. Ollero, J.M.D.D., B.C.: Arre: Integrated systems for early forest-rire detection. III International Conference on Forest Fire Research 2 (1998) 1977-1988

8. Sahin, Y.G.: Animals as mobile biological sensors for forest fire detection. Sensors 7 (2007) 3084-3099

9. Florent Lafarge, X.D.y.J.Z.: Forest fire detection based on gaussian field analysis. European Signal Processing Conference (EU-SIPCO) (2007)

10. González Becerra Diego Alexander, Hernández Velázquez Xochitl, J.R.I.: Simulador de incendio forestal(sif). Benemérita Universidad Autónoma de Puebla (2007)

11. Seng Chuan TAY, Wynne HSU, K.H.L.: Spatial data mining: Clustering of hot spots and pattern recognition. In: IEEE International Geo-science and Remote Sensing Symposium. Volume 6. (2003) 3685-3687

12. Daniel Santin Gonzalez, C.P.L.: Mineria de datos: Tecnicas y Herramientas. Thompson, Madrid (2007)

13. Martin del Brío, Bonifacio; Sanz, A.: Redes Neuronales y Sistemas Borrosos. AlfaOmega, México (2007) 